\title{
Causing a commotion
}

\section{Niche construction: do the changes that organisms make to their habitats transform evolution and influence natural selection?}

\section{Kevin N. Laland, John Odling-Smee} and Marcus W. Feldman

$\square$ volutionary biology has always generated vigorous debate, from the arguments about lamarckian inheritance during the 1900s, to the more recent disputes over punctuated equilibria and group selection. The latest spat — a storm in a teacup compared with these earlier controversies - concerns the seemingly prosaic observation that the activities of organisms bring about changes in their environments - a process known as niche construction. In January this year, ecologist John Vandermeer described the niche-construction perspective as "a major breakthrough", yet an earlier review by Laurent Keller in Nature (425, 769-770; 2003) dismissed it as hyperbole, and evolutionary biologist Richard Dawkins warns of its "pernicious" reasoning in a forthcoming article. It is a subject that typically provokes strong and polarized responses. What is the fuss about?

At the heart of the controversy lies the nature of causality in evolution. Adaptation is conventionally seen as a process by which natural selection shapes organisms to fit preestablished environmental 'templates'. The causal arrow points in one direction only: it is environments, the source of selection, that determine the features of living creatures.

Yet it is also obvious that organisms bring about changes in environments. Numerous animals manufacture nests, burrows, holes, webs and pupal cases. Plants change the levels of atmospheric gases and modify nutrient cycles. Fungi decompose organic matter, and bacteria engage in decomposition and nutrient fixation. The standard view of evolution does not deny this, but treats niche construction as no more than the product of selection.

Conversely, from the niche-construction perspective, evolution is based on networks of causation and feedback. Organisms drive environmental change and organismmodified environments subsequently select organisms. The argument that niche construction does not play a causal role in evolution because it is partly a product of natural selection, makes no more sense than would the counter-proposal that natural selection can be disregarded because it is partly a product of niche construction.

At present, standard evolutionary theory models the evolutionary consequences of niche construction solely in terms of fitness 'pay-offs' to the genes expressed in niche construction. For us, this is unsatisfactory. First, it

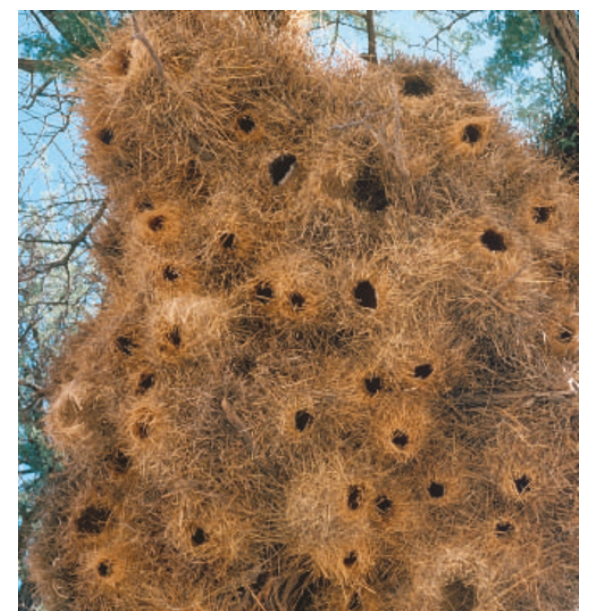

Networking: do birds' nests shape evolution?

misses part of the causal story. When a beaver builds a dam it not only affects the propagation of dam-building genes, but it must also transform the selection acting on a host of other beaver traits. Second, some organism-driven changes in the environment persist as a legacy to modify selection on subsequent generations - an ecological inheritance. Contemporary earthworms are adapting to a soil environment largely constructed by their ancestors. Third, genes often exert a weak regulatory influence on niche construction. There are no 'genes for' dairy farming; nor is it (strictly) an adaptation, but rather an adaptive cultural practice. Yet this activity has generated selection that favours genes for lactose absorption.

The niche-construction perspective was brought to the attention of evolutionarybiologists by Richard Lewontin in the 1980s. The classic view of evolution, he said, can be encapsulated in the pair of differential equations $O^{\prime}=f(O, E)$ and $E^{\prime}=g(E)$, where the primes indicate differentiation with respect to time, the state variables are the organism $(O)$ and environment $(E)$, and the function $f$ stipulates evolution ( $g$, the function describing environmental change, is considered beyond the remit of evolutionary biology). Lewontin claimed a more accurate formulation to be $O^{\prime}=f(O, E)$ and $E^{\prime}=g(O, E)$, because part of the cause of evolution is the impact of the organism on its environment. Yet his seminal essays had little immediate impact. What has changed?

First, accumulated empirical evidence for niche construction, in everything from ants to boreal forests, means that there is no longer any dispute over its ubiquity. The view of leading evolutionist Theodosius Dobzhansky that "man alone adapts himself ... by actively or even deliberately changing the environment" is no longer tenable. Niche construction is not the prerogative of large populations, keystone species or clever animals - it is a fact of life.

Second, recent mathematical theory has established that niche construction changes the evolutionary dynamic. Niche construction can create new equilibria, affect the stability of others, generate unusual phenomena, such as momentum effects (where populations continue to evolve in the same direction after selection has stopped or reversed) and inertia effects (a delayed evolutionary response to selection), as well as opposite and catastrophic responses to selection.

Third, and most importantly, empirical methods have been devised that allow evolutionary biologists and ecologists to ask — and answer - new questions and shed fresh light on old ones. For instance, evolutionary biologists can use genomics or proteomics techniques to track the evolutionary consequences of niche construction in microorganisms such as bacteria. And ecologists can make predictions about which species will invade a community and whether or not the invasion will be benign, based on knowledge of the invader's tolerance to the residents' niche construction, and the nature of the invader's activities.

Already, niche construction is stimulating palaeobiologists and ecologists to organize conferences and symposia, philosophers to commission special editions of journals and researchers as diverse as archaeologists and primatologists to grow excited by its ideas and methods. Ultimately, it is here that the fate of the niche-construction 'school' will be decided. If this early enthusiasm proves a fleeting fad, and there is little genuine utility in the alternative panorama, it will fade. However, if researchers continue to start using these new tools then, in the words of philosopher David Hull: "the result should be a massive reorientation of evolutionary theory".

Kevin N. Laland is in the School of Biology, St Andrews University, UK. John Odling-Smee is at the Institute of Biological Anthropology, Oxford University, UK. Marcus W. Feldman is in the Department of Biological Sciences, Stanford University, USA.

\section{FURTHER READING}

Jones C. G. et al. Ecology 78, 1946-1957 (1997). Laland K. N., Odling-Smee J. \& Feldman M. W. Proc. Natl Acad. Sci. USA 96, 10242-10247 (1999). Odling-Smee J., Laland K. N. \& Feldman M. W. Niche Construction. The Neglected Process in Evolution. Monographs in Population Biology 37 (Princeton Univ. Press, Princeton, 2003).

Vandermeer J. Science 303, 472-474 (2004).

www.nicheconstruction.com 